\title{
Dynamic visual acuity: Normative functions and practical implications
}

\author{
GERALD M. LONG and DAVID L. PENN \\ Villanova University, Villanova, Pennsylvania
}

\begin{abstract}
The dynamic visual acuity (DVA) of 52 college students was determined for three target velocities $\left(60^{\circ}, 90^{\circ}\right.$, and $120^{\circ}$ per sec) at each of two target durations and two luminance levels. Although all observers were prescreened for 20/20 acuity, widely differing DVA scores were found across observers under identical stimulus conditions. No relationship between DVA scores and contrast sensitivity scores was revealed. The often overlooked role of DVA in everyday activities is reiterated, and the potential utility of the normative DVA function obtained in this work is discussed.
\end{abstract}

In the past 40 years there has been sporadic interest in the assessment of a particular aspect of visual functioning that is generally overlooked by traditional measures, and yet may play a critical role in many real-world activities. This visual capability is known as dynamic visual acuity (DVA); the term refers to the ability of an observer to resolve fine detail in an object when there is relative motion between the observer and that object. The classic finding in numerous DVA studies has been that increasing the angular velocity of an object beyond a rather low value results in a positively accelerating degradation in acuity, so that even at rather low speeds of $30^{\circ}-40^{\circ}$ per sec, threshold for detail can be several times larger than that found for a stationary target (see Miller \& Ludvigh, 1962, and Morrison, 1980, for reviews).

The practical utility of DVA assessment is underscored by reference to two basic findings. First, individuals possessing identical static visual acuity as traditionally measured by standard eye charts (e.g., Snellen letters or Landolt $\mathrm{C}$ targets) can vary considerably among themselves on a DVA task (e.g., Goodson \& Morrison, 1980; Ludvigh \& Miller, 1958). In other words, a person's ability to resolve high-speed targets is not predictable from his/her static acuity (e.g., Fergenson \& Suzansky, 1973). Second, there is some evidence that performance on certain practical tasks, such as flying, driving, and athletics, may be significantly related to DVA. For example, it has been found that the DVA of pilots contributes to their performance on various flying tests to a greater degree than does their static acuity (DeKlerk, Eernst, \& Hoogerheide, 1964). DVA has also been reported to have a stronger relationship to driving performance than does any other standard vision test examined (e.g., Henderson \& Burg, 1973). The implications for driving safety are obvious: individuals whose standard (i.e., static) acuity has met the vision criterion for obtaining a driver's license may significantly differ from one another in their ability to resolve objects once the vehicle is in motion. This clearly

\footnotetext{
This research was supported in part by Grant 1-R03-EY05846-01 from the National Eye Institute to the first author. The authors' address is Department of Psychology, Villanova University, Villanova, PA 19085.
}

suggests that our vision assessment procedure for licensing drivers is incomplete. There appears to be good reason to accept a critical role of DVA in various athletic activities (e.g., Sherman, 1980; Stine, Arterburn, \& Stern, 1982). Specifically, DVA has been implicated in the ability to catch a ball (Sanderson \& Whiting, 1974), in batting averages among Little Leaguers (Falkowitz \& Mendel, 1977), and in basketball shooting accuracy (Morris \& Kreighbaum, 1977).

The DVA work described in this article had two basic goals. The first was to obtain normative DVA data across a representative range of viewing conditions with a sample of college-aged observers prescreened for normal (20/20) static acuity. If DVA is to be used for practical visual assessment, then a normative data base is essential. To obtain a representative range of DVA performance, we exposed subjects to three levels of target angular velocity $\left(60^{\circ}, 90^{\circ}\right.$, and $120^{\circ}$ per sec), two levels of target duration ( 250 and $400 \mathrm{msec})$, and two levels of target luminance $\left(35\right.$ and $\left.160 \mathrm{~cd} / \mathrm{m}^{2}\right)$. The three levels of target velocity bracket the typical velocities employed in this type of work (e.g., Goodson \& Morrison, 1980). The longer duration $(400 \mathrm{msec})$ matches that used in previous work; this duration theoretically permits both an initial saccade and a subsequent smooth-pursuit eye movement (Miller \& Ludvigh, 1962). The 250-msec value was chosen in order to restrict task performance to a single saccadic eye movement alone; eye movement recording research indicates that the saccade should be complete by $250 \mathrm{msec}$ (Brown, 1972b). The two luminance levels were selected to assess the reported hypersensitivity of DVA to energy manipulations (see, e.g., Morrison, 1980). Although the luminance-acuity function for static targets asymptotes by about $10 \mathrm{fL}$ (e.g., Riggs, 1965), DVA has been reported to continue to improve at levels far beyond this (e.g., Miller, 1958). Hence, our inclusion of the two luminance levels allows us to replicate this finding over a range of DVA viewing conditions.

The second goal of the present work was to gain better insight into the visual processes underlying DVA. This was accomplished in part by use of the range of stimulus 
conditions described above. For example, the choice of the two target durations to separate saccadic accuracy from the total DVA setting also permitted a separate determination of luminance effects on saccadic performance in a DVA context, as well as on the more typical DVA task. In addition, we hypothesized that the different eye movement systems (i.e., saccadic and pursuit) involved in DVA might exhibit different degrees of dependence on the "transient" and "sustained" neural channels. An impressive amount of psychophysical and neurophysiological research (see Regan, 1982, for a recent review) indicates that the human visual system comprises parallel channels that are selectively sensitive to different characteristics of a visual stimulus. The application of the multiplechannel theory of vision has hitherto not been applied to DVA, although certain relationships appear to be quite likely. For example, the transient channels alone project to the midbrain structures (Rodiek, 1979), and these structures are known to play a critical role in saccadic eye movements (e.g., Leigh \& Zee, 1983). Hence, under DVA viewing conditions in which the saccadic eye movements have the dominant role (e.g., 250-msec duration and high target velocity), the input from the transient channels would be most critical. On the other hand, if the pursuit eye movements, which some have suggested are dominated by more cortical centers (Leigh \& Zee, 1983), play a relatively more important role under other viewing conditions (e.g., 400-msec duration and low target velocities), the input from the sustained channels would have greater relevance to DVA performance. Consequently, in the present work each observer's contrast sensitivity was determined over a range of spatial frequencies, and these scores were then correlated with DVA under the wide range of viewing conditions employed.

\section{METHOD}

\section{Subjects}

Fifty-two subjects participated in partial fulfillment of a course requirement in general psychology at Villanova University. Each subject's static acuity was assessed using a Titmus vision tester; only those individuals possessing a minimum of $20 / 20$ vision (corrected or uncorrected) were allowed to participate. Individuals who wore tinted glasses or contacts were excluded. Each individual participated in two 60-min sessions that were held 4 to 10 days apart.

\section{Apparatus and Procedure}

The first part of Session 1 involved the assessment of subjects' contrast sensitivity. Contrast sensitivity measurements were obtained using a Nicolet CS 2000 Contrast Sensitivity System, which is the same basic system described elsewhere in the literature (Ginsburg et al., 1984; Long \& Penn, 1987). Vertical sine-wave gratings were viewed binocularly by an observer seated $3 \mathrm{~m}$ from a display monitor, which subtended a visual angle of about $4^{\circ} \times 5^{\circ}$. The spatial frequency and contrast of the gratings were controlled by a microprocessor. The sequence of the gratings presented with a test session and the analysis of the observer's response were also under control of the microprocessor within a standardized test format (see below), All gratings had a mean luminance of $100 \mathrm{~cd} / \mathrm{m}^{2}$, which was calibrated daily.

Within the "standard test" format described by Nicolet, each observer received two practice trials with stationary gratings of 0.5 and $6.0 \mathrm{cpd}$. Six test trials then followed at spatial frequencies of $0.5,1.0,3.0,6.0$, 11.4 , and $22.8 \mathrm{cpd}$. Each trial began with a preview of the spatial frequency to be examined on that trial. A pair of tones signaled the start of the preview period, which consisted of a 1-sec increase in the contrast of that spatial frequency from zero to $50 \%$. The $50 \%$ contrast level was displayed for $2 \mathrm{sec}$, after which the contrast decreased to zero. A single tone then warned the observer of the start of the actual test trial, which employed a Békésy tracking procedure. Essentially, the subject was instructed to depress the response button whenever a grating was visible and to release the button whenever the grating disappeared. The microprocessor automatically decreased grating contrast when the button was depressed and increased grating contrast when the button was released. After four button presses and four button releases, a tone signaled the end of the trial; the microprocessor then computed the mean and standard deviation of the obtained contrast threshold and printed these data before proceeding to the next trial.

In the second half of Session 1 the subjects were familiarized with and then practiced the basic DVA task. The subjects were seated directly under the center of a rotating front-surface mirror, which was mounted vertically on a variable-speed turntable. A variable-size Landolt C target (positive contrast) was projected onto the mirror and then reflected on a white hemicylindrical screen viewed by the observer at a constant distance of $1.5 \mathrm{~m}$. The target always appeared initially $15^{\circ}$ to the right of fixation and moved in a left-to-right horizontal direction. The speed of the turntable controlled angular velocity of the reflected target for the three levels of target speed. A Gerbrands millisecond shutter mounted on the lens of the Kodak Ektagraphic projector controlled target duration at either 250 or $400 \mathrm{msec}$. Finally, Kodak No. 96 neutral density filters placed in front of the shutter controlled target luminance at either 11 or $50 \mathrm{fL}\left(35\right.$ or $\left.160 \mathrm{~cd} / \mathrm{m}^{2}\right)$. Background luminance of the screen was constant at $10 \mathrm{fL}\left(32 \mathrm{~cd} / \mathrm{m}^{2}\right)$.

Subjects were exposed to four practice sets of stimuli: these sets consisted of the stimuli presented at the two extreme target speeds $\left(60^{\circ}\right.$ and $120^{\circ}$ per sec) and two target durations (250 and $400 \mathrm{msec}$ ). For these practice trials, the targets were maintained at the high luminance level. For each DVA run, subjects viewed a series of Landolt-C targets decreasing in size from about $80^{\prime}$ of arc to a minimum of about $8^{\prime}$ of arc until they were at threshold. Following the general procedure of other investigators (e.g., Goodson \& Morrison, 1980), the size of the Landolt- $C$ was reduced from one trial to the next until the subject made an error in reporting the location of the gap in the Landolt- $\mathrm{C}$ (upper left, upper right, lower left, or lower right). Target size was then increased to the previous size, and, if correctly identified, reduced again. Two errors for a given sized target were necessary to terminate the run; the next largest target size was defined as the threshold for that run.

In Session 2, subjects practiced the DVA task under the same stimulus conditions as were employed in the first practice session. The subjects then received all 12 stimulus conditions ( 3 angular velocities, 2 target durations, and 2 target luminance levels), uniquely randomized for each subject.

\section{RESULTS AND DISCUSSION}

Mean acuity threshold (in minutes of arc) for the 52 observers at two target durations as a function of target speed is depicted in Figure 1. For reasons discussed below, this figure presents the results from only the lowluminance condition. A 3 (target angular velocity) $\times 2$ (target luminance level) $\times 2$ (target duration) withinsubjects analysis of variance revealed significant main effects of target duration $[F(1,51)=29.83, p<.0001]$, target angular velocity $[F(2,102)=79.23, p<.0001]$, and target luminance $[F(1,51)=200.58, p<.001]$. The following interactions were significant: target luminance $\times$ target duration $[F(1,51)=13.08, p<.001]$ and target luminance $\times$ target angular velocity $[F(2,102)=$ $14.83, p<.0001]$. It should be noted, however, that interpretation of the interactions and the luminance main effect must be tempered by the fact that subjects tended to "bottom out" (i.e., resolve the smallest Landolt C 


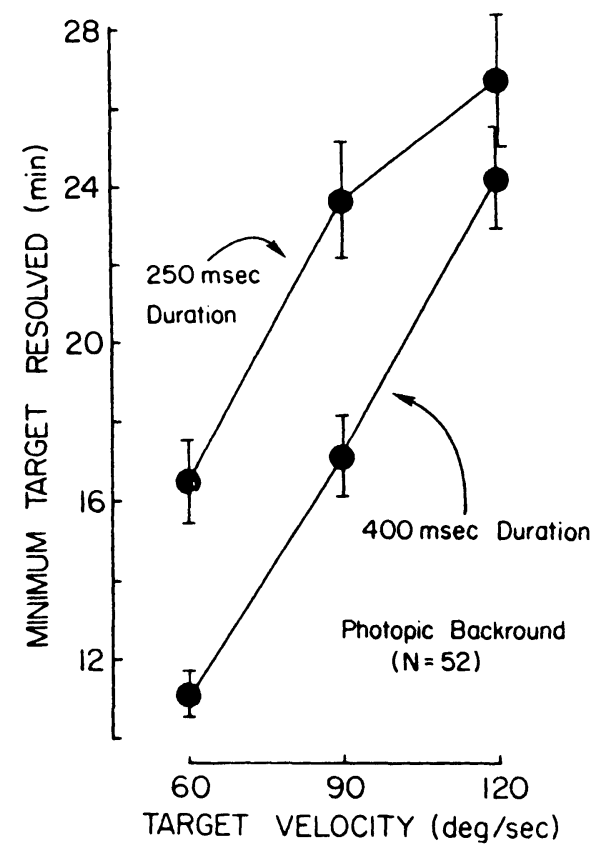

Figure 1. Mean target resolution threshold (in minutes of arc) for 52 observers as a function of target velocity at two target durations. Target luminance was held constant at $35 \mathrm{~cd} / \mathrm{m}^{2}$.

presented) at the high luminance level. This indicates that DVA performance at this luminance level cannot be reliably assessed by our apparatus because of the limited range of target sizes available.

Individual differences in DVA performance are illustrated in Figure 2, which depicts acuity threshold as a function of target angular velocity for 4 subjects at one duration $(400 \mathrm{msec})$ and a constant luminance level. This figure demonstrates that these 4 relatively practiced subjects, prescreened for 20/20 static acuity, varied considerably in their performance on the DVA task.

The results shown in Figures 1 and 2 clearly demonstrate that our apparatus and procedures are able to replicate traditional DVA findings. Increasing target angular velocity had a marked effect on DVA, and decreasing either target luminance level or target duration engendered further decreases in this type of acuity. And very importantly, as in previous research (e.g., Goodson \& Morrison, 1980), individual differences on the DVA task among observers prescreened for equivalent 20/20 acuity were manifest.

The present findings also address conclusions made in a recent article on smooth eye-pursuit velocity by Meyer, Lasker, and Robinson (1985). In that study, it was demonstrated that the smooth-pursuit eye movement system can match target speeds up to approximately $90^{\circ}-100^{\circ}$ per sec. However, the present DVA findings might appear to be somewhat discrepant with these results in that we found a marked decline in performance at velocities for which Meyer et al. reported excellent pursuit gain. Thus, the conclusion that smooth pursuit can match target speed up to rather high levels must be viewed with caution; it may apply only to optimal conditions.
To assess the hypothesized role of sustained and transient channel activity in DVA, we computed Pearson's $r$ between DVA and contrast sensitivity scores across the entire range of stimulus conditions examined. As noted earlier, because of the "cellar" effect (i.e., minimal subject variance) at the high luminance level, only DVA scores from the low luminance level were used in this correlational analysis. Nonetheless, inspection of the intercorrelation matrix revealed only nonsignificant correlations $(p>.05)$. Because there was some indication of positive skewness, the data were reanalyzed following a log transform of the data; correlations were still nonsignificant. However, it should be noted that subsequent work from this lab (Long \& Tuck, 1986) has revealed that, at least for nonpracticed subjects, the von Békésy method for determining contrast sensitivity has disappointingly low reliability. This, in turn, suggests that the lack of relationship between DVA and CSF may result from low reliability in the CSF measure.

\section{CONCLUSIONS}

The present results have the following implications for DVA assessment. First, the results appear to buttress the validity of the present study's apparatus and procedures for the rapid assessment of DVA performance. The single area of weakness revealed by this work appeared to be the minimum target size available $\left(8^{\prime}\right.$ of arc), which was insufficient for threshold determination under some stimulus conditions. With modification, this lower limit has since been reduced to $3^{\prime}$ of arc. A series of DVA

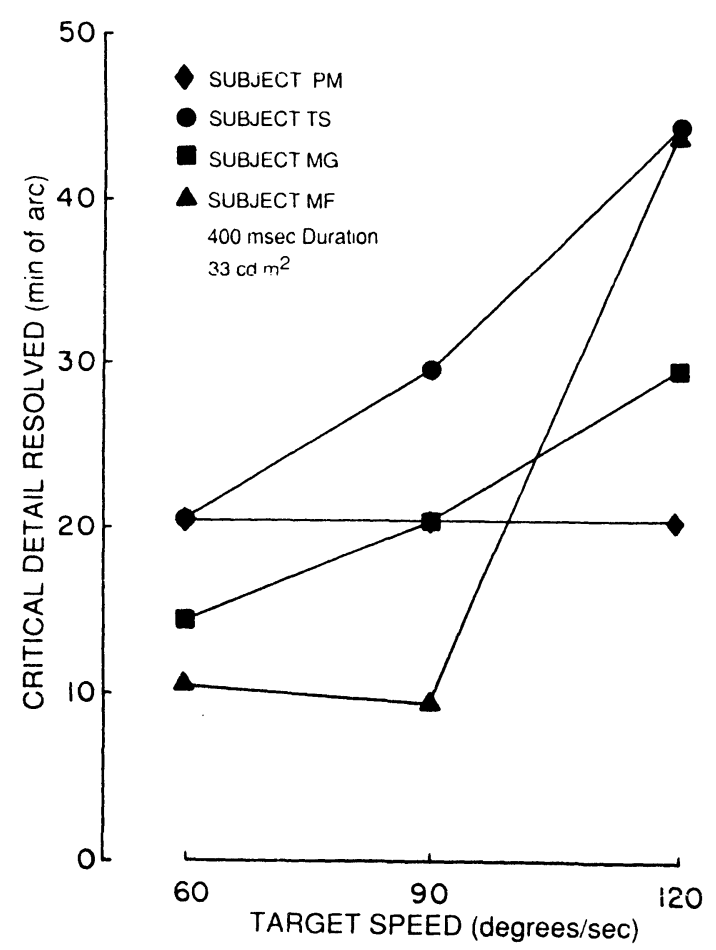

Figure 2. Target resolution threshold (in minutes of arc) for $4 \mathrm{ob}-$ servers as a function of target velocity. Target luminance and target duration were held constant. 
studies with this apparatus and procedures is now under way (e.g., Garvey \& Long, 1986). Second, this investigation was able to replicate the oft-cited finding that individuals with identical static acuity vary considerably in their performance on a DVA task. Under identical DVA conditions, it was not unusual to find some subjects' threshold targets to be several times larger than those of other subjects, even though all subjects had been prescreened for 20/20 static acuity. Third, normative DVA data were obtained on a population prescreened for normal static acuity. This is an obvious first step in the process of standardizing DVA assessment as a potential visual screening procedure. Fourth, concerning future DVA research, the marked sensitivity of DVA to target luminance suggests that related energy factors, such as background luminance level and target color, may individually and/or interactively affect DVA performance in some interesting ways. Unfortunately, unambiguous interpretation of the luminance effect was not possible in the present study because of the obvious cellar effect obtained at the high luminance level. Nevertheless, the extreme sensitivity of DVA to target energy may have some bearing on very practical questions concerning DVA and visual performance, such as whether the relationship between DVA and driving ability differs under photopic (daylight) and scotopic (nighttime) driving conditions, or whether traffic signs of a particular color are easier to read than others when viewed from a moving vehicle (Garvey \& Long, 1986). Fifth, due to the low reliability of the particular CSF measure used, the hypothesized relationship between DVA and parallel visual channels must still be considered undetermined. This strongly suggests that our original hypothesis should be reassessed with a more reliable method for determining an individual's contrast sensitivity. Subsequent findings (Long \& Tuck, 1987) suggest that the method of adjustment would be a more reliable measure of CSF, and a new study using this alternate CSF method is planned. Finally, the discrepancy between our findings and those of Meyer et al. (1985) suggests the need for greater precision in the language employed in the DVA/eye-movement literature. For example, good eye/target-velocity correspondence, which is what Meyer et al. measured, does not necessarily indicate good eye/target-position correspondence (i.e., Brown, 1972a). Moreover, the conditions used by Meyer et al. were optimized for pursuit eye movement. The vast majority of eye movement studies report that pursuit gain falls off considerably at velocities beyond $20^{\circ}-30^{\circ}$ per sec (e.g., Collewijn \& Tamminga, 1984; Leigh \& Zee, 1983). Hence, the degree to which Meyer et al.'s findings can be generalized is unknown. This point becomes all the more relevant when we consider that DVA reflects the final output of several interacting visual processes, including saccadic eye movements, pursuit eye movements, and visual optics.

\section{REFERENCES}

Brown, B. (1972a). The effect of target contrast variation on dynamic visual acuity and eye movements. Vision Research, 12, 1213-1224.

Brown, B. (1972b). Resolution thresholds for moving targets at the fovea and in the peripheral retina. Vision Research, 12, 293-304.

Collewijn, H., \& TAmminga, E. P. (1984). Human smooth and saccadic eye movements during voluntary pursuit of different target motions on different backgrounds. Journal of Physiology, 351, 217-250.

DeKlerk, L. F., Eernst, J., \& HoOgerheide, J. (1964). The dynamic visual acuity of 30 selected pilots. Aeromedica Acta, 9, 129-136.

Falkowitz, C., \& MENDEL, H. (1977). The role of visual skills in batting averages. Optometric Weekly, 68, 42-57.

Fergenson, P. E., \& Suzansky, J. W. (1973). An investigaton of dynamic and static visual acuity. Perception, 2, 246-256.

GARveY, P. M., \& LoNG, G. M. (1986, October). Dynamic visual acuity with colored targets. Paper presented at the Fifth Annual Adelphi University Applied Experimental Psychology Conference, Adelphi, NY.

Goodson, J. E., \& MorRISON, T. R. (1980). Stimulus determinants of dynamic visual acuity: I. Background and exploratory data (NAMRL1270). Pensacola, FL: Naval Aerospace Medical Research Laboratory.

Henderson, R. L., \& BURG, A. (1973). The role of vision and audition in truck and bus drivers (Rm-(1)-5260/000/00). Santa Monica, CA: Systems Development Corp.

LEIGH, R. J., \& ZEE, D. S. (1983). The neurology of eye movements. Philadelphia: F. A. Davis.

LONG, G. M, \& PENN, D. L. (in press). Normative contrast sensitivity functions: The problem of comparison. American Journal of Optometry \& Physiological Optics.

Long, G. M., \& TuCK, J. P. (1986, November). Reliabilities of various measures of contrast sensitivity functions. Paper presented at the annual meeting of the Psychonomic Society, New Orleans.

LoNG, G. M., \& TuCK, J. P. (1987). Reliabilities of alternate measures of contrast sensitivity functions. Manuscript in preparation

Ludvigh, E., \& Miller, J. W. (1958). Study of visual acuity during the ocular pursuit of moving test objects: I. Introducton. Journal of the Optical Society of America, 48, 799-802.

Meyer, C. H., Lasker, A. G., \& Robinson, D. A. (1985). The upper limit of human smooth pursuit velocity. Vision Research, 25, 561-563.

MilleR, J. W. (1958). Study of visual acuity during the ocular pursuit of moving test objects: II. Effects of direction of movement, relative movement, and illumination. Journal of the Optical Society of America, 48, 803-808.

Miller, J. W., \& Ludvigh, E. (1962). The effect of relative motion on visual acuity. Survey of Ophthalmology, 7, 83-116.

Morris, G. S., \& Kreighbaum, E. (1977). Dynamic visual acuity of varsity women volleyball and basketball players. Research Quarterly of the American Association of Health \& Physical Education, 48, 480-483.

MORRISON, T. R. (1980). A review of dynamic visual acuity (NAMRL Monograph No. 28). Pensacola, FL: Naval Aerospace Medical Research Laboratory.

REGAN, D. (1982). Visual information channelling in normal and disordered vision. Psychological Review, 89, 407-444.

RigGs, L. A. (1965). Visual acuity. In C. H. Graham (Ed.), Vision and visual perception (pp. 321-349). New York: Wiley.

Rodiek, R. W. (1979). Visual pathways. Annual Review of Neuroscience, 2, 193-225.

Sanderson, F. H., \& Whiting, H. T. A. (1974). Dynamic visual acuity and performance in a catching task. Journal of Motor Behavior, 6, 87-94.

ShERMAN, A. (1980). Overview of research information regarding vision and sports. Journal of the American Optometric Association, 51, 661-665.

Stine, D., Arterburn, M. R., \& Stern, N. S. (1982). Vision and sports: A review of the literature. Journal of the American Optometric Association, 53, 627-633. 